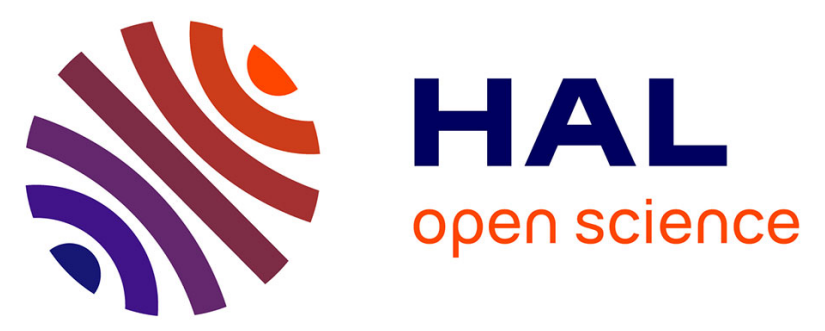

\title{
Phenotype heterogeneity in cancer cell populations
}

Luis Almeida, Rebecca H. Chisholm, Jean Clairambault, Alexandre

Escargueil, Tommaso Lorenzi, Alexander Lorz, Emmanuel Trélat

\section{To cite this version:}

Luis Almeida, Rebecca H. Chisholm, Jean Clairambault, Alexandre Escargueil, Tommaso Lorenzi, et al.. Phenotype heterogeneity in cancer cell populations. ICNAAM 2015 Session 70: "Mathematical models and methods to investigate heterogeneity in cell and cell population biology", Jean Clairambault, Sep 2015, Rhodes, Greece. hal-01249242

\section{HAL Id: hal-01249242 \\ https://hal.inria.fr/hal-01249242}

Submitted on 30 Dec 2015

HAL is a multi-disciplinary open access archive for the deposit and dissemination of scientific research documents, whether they are published or not. The documents may come from teaching and research institutions in France or abroad, or from public or private research centers.
L'archive ouverte pluridisciplinaire HAL, est destinée au dépôt et à la diffusion de documents scientifiques de niveau recherche, publiés ou non, émanant des établissements d'enseignement et de recherche français ou étrangers, des laboratoires publics ou privés. 


\title{
Phenotype heterogeneity in cancer cell populations
}

\section{Luis Almeida*, Rebecca Chisholm ${ }^{\dagger}$, Jean Clairambault**, Alexandre Escargueil ${ }^{\ddagger}$, Tommaso Lorenzi ${ }^{\S}$, Alexander Lorz ${ }^{\mathbb{I}}$ and Emmanuel Trélat ${ }^{\|}$}

\author{
*CNRS UMR 7598, LJLL, \& INRIA MAMBA team, Sorbonne Universités, UPMC Univ Paris 06, Boîte courrier 187, 4 Pl. \\ Jussieu, 75252 Paris cedex 05, France,luis@ann.jussieu.fr \\ $\dagger$ School of Biotechnology and Biomolecular Sciences, University of New South Wales, Sydney, Australia, \\ rebecca.chisholm@gmail.com \\ ** INRIA MAMBA team \& LJLL, UMR 7598, Sorbonne Universités, UPMC Univ Paris 06, Boîte courrier 187, 4 Pl. Jussieu, \\ 75252 Paris cedex 05, France, jean.clairambault@inria.fr, corresponding author \\ ¥INSERM “Cancer Biology and Therapeutics”, Sorbonne Universités, UPMC Univ Paris 06, UMR_S 938, CDR St Antoine, \\ Hôpital St Antoine, 184 Fbg. St Antoine, 75571 Paris cedex 12, France, alexandre.escargueil@upmc.fr \\ ${ }^{\S}$ CMLA, ENS Cachan, 61, Av. du Président Wilson, 94230 Cachan cedex \& INRIA MAMBA team, \& LJLL, UMR 7598, \\ UPMC Univ Paris 06, Boîte courrier 187, 4 Pl. Jussieu, 75252 Paris cedex 05, France, tommaso.lorenzi@gmail.com \\ ${ }^{I}$ Sorbonne Universités, UPMC Univ Paris 06, LJLL, UMR 7598 \& INRIA Boîte courrier 187, 4 Pl. Jussieu, 75252 Paris \\ cedex 05, France, alex.lorz@ann.jussieu.fr \\ Institut Universitaire de France, Sorbonne Universités, UPMC Univ Paris 06, LJLL, UMR 7598, Boîte courrier 187, UPMC \\ Univ Paris 06, 4 Pl. Jussieu, 75252 Paris cedex 05, France, emmanuel.trelat@upmc.fr
}

\begin{abstract}
Phenotype heterogeneity in cancer cell populations, be it of genetic, epigenetic or stochastic origin, has been identified as a main source of resistance to drug treatments and a major source of therapeutic failures in cancers. The molecular mechanisms of drug resistance are partly understood at the single cell level (e.g., overexpression of ABC transporters or of detoxication enzymes), but poorly predictable in tumours, where they are hypothesised to rely on heterogeneity at the cell population scale, which is thus the right level to describe cancer growth and optimise its control by therapeutic strategies in the clinic. We review a few results from the biological literature on the subject, and from mathematical models that have been published to predict and control evolution towards drug resistance in cancer cell populations.

We propose, based on the latter, optimisation strategies of combined treatments to limit emergence of drug resistance to cytotoxic drugs in cancer cell populations, in the monoclonal situation, which limited as it is still retains consistent features of cell population heterogeneity. The polyclonal situation, that may be understood as "bet hedging" of the tumour, thus protecting itself from different sources of drug insults, may lie beyond such strategies and will need further developments. In the monoclonal situation, we have designed an optimised therapeutic strategy relying on a scheduled combination of cytotoxic and cytostatic treatments that can be adapted to different situations of cancer treatments.

Finally, we review arguments for biological theoretical frameworks proposed at different time and development scales, the so-called atavistic model (diachronic view relying on Darwinian genotype selection in the coursof billions of years) and the Waddington-like epigenetic landscape endowed with evolutionary quasi-potential (synchronic view relying on Lamarckian phenotype instruction of a given genome by reversible mechanisms), to represent evolution towards heterogeneity, possibly polyclonal, in cancer cell populations and propose innovative directions for therapeutic strategies based on such frameworks.
\end{abstract}

Keywords: Cell population dynamics, Partial differential equations, Physiologically structured models, Evolution, Cancer, Drug resistance PACS: 87.17.Aa, 87.17.Ee

\section{Biological background and hypotheses}

Drug resistance: the main medical issue of oncology. The importance of drug-induced drug resistance in cancer cell populations has long been recognised in the clinic, its molecular mechanisms partially identified in vitro, and mathematical models proposed to account for it and predict it [1]. Undisputable cures have been obtained in some occurrences by targeted therapies when a unique target exists and a specified molecule has been identified, able to successfully inhibit it (imatinib in chronic myelogenous leukaemia or all-transretinoic acid in acute promyelocytic leukaemia), or by combinations of cytotoxic drug in cases of solid tumours with intact p53 [2]. However, most cancers, after initial shrinkage of tumour mass and temporary remission due to chemotherapy, with variable delays relapse and eventually escape all therapies. This is due to drug-induced resistance in cancer cell populations, a phenomenon for which different hypotheses have been put forward to explain its mechanisms.

The simplest of these proposed mechanisms is mutation: a subclone of the cancer cell population encompasses by chance a mutation that makes it amenable to tolerance to the drug, and Darwinian "survival of the fittest" makes the 
rest. This is the case in many occurrences of cancers, and it has been well documented in acute myeloblastic leukaemias [3]. However, possible reversal to total sensitivity after drug withdrawal [4] excludes mutation and Darwinian selection from being the only mechanism by which drug-induced drug resistance emerges in cancer cell populations.

Furthermore, it has been shown in [4] that epigenetic enzymes may be essential to the occurrence of drug-tolerant cancer cell populations, a mechanism that may be related to their characteristic phenotypic plasticity, without necessary somatic mutations, and without necessarily either assuming the existence of so-called "cancer stem cells". Rather, one may propose that deregulation of epigenetic mechanisms (either spontaneous, under drug environmental pressure, or also possibly due to fine 'epimutations' such as of the TET2 gene [5]), clinically undetectable for a very long time as long as normal cell differentiation, proliferation and apoptosis mechanisms are not affected, may allow a tumour by DNA/histone methylations or demethylations of a limited number of its cells, e.g., to silence tumour suppressor genes or reveal physiologically silenced oncogenes. Such deregulated epigenetic mechanisms are likely to constitute the molecular basis of this phenotype adaptation that is also qualified cell plasticity.

Phenotype heterogeneity. In cancer cell populations, heterogeneity in motility (EMT), in plasticity with respect to differentiation status, or in response to anti-cancer drugs, may certainly depend on the genetic status of cell clones presenting different mutations [6], but experiments on genetically homogeneous cancer cell lines have also shown that under drug pressure, different phenotypes identifiable with respect to proliferation and survival or visible as stained by membrane antigens may be present, appear and disappear [4]. Such fast changes in phenotype, adaptations to stress, not due to mutations since they may be completely reversible, rely - when they do not result from mere local regulations - on epigenetic changes ('epimutations'), due to fixation on the DNA or on histones of methyl or acetyl radicals that silence specific genes. Such epigenetic mechanisms, that are at the basis of all cell differentiation, also account for plasticity, i.e., fast phenotype changes, in cancer cell populations without - or before - the occurrence of any mutation. Determining whether such phenotype differences in genetically homogeneous cell populations are due to epigenetic mechanisms or to pure stochastic events is still most of the times an open question.

Need for innovative therapeutic strategies in cancer. Drug resistance occurs in all fields of medicine in which populations of living cells, viruses or parasites, in other words all proliferating populations of organisms that reproduce in the human body, are concerned. This is particular true for cancer cell populations, that are endowed with the rich mammalian genome, and are amenable to high phenotypic plasticity [7, 8]. Remarkably targeted on precise intracellular pathways though they may be, targeted therapies with few exceptions (in particular imatinib in CML [9] and ATRA in APL [6]), after some initial success, generally induce resistance to their administration in cancer cell populations. Note that such resistance is most often reversible, so that some clinical teams practice a so-called "drug holiday", so as not to induce actual drug resistance, or reduce it to minimum. As we show below, it is also possible, in a strategy of combination of cytostatic and cytotoxic drugs, to leave the administration of cytotoxic drugs (which, as life-threatening for a tumour, are those that most induce resistance) for the very last moment, thus avoiding in both cases "instruction" of the cancer cell population during prolonged durations towards drug resistance.

\section{Physiologically structured population models}

Continuous models of drug resistance: adaptive dynamics. We consider the problem of adaptation of a resistance phenotype $x \in[0,1]$ to a given drug expressed by healthy and cancer cells, with high plasticity in the case of cancer cells. Such plasticicity is determined by the effects of drugs on the proliferation and death rates in the two populations. Our basic model, in which $n_{H}(t, x)$ and $n_{C}(t, x)$ represent densities in a given tissue of healthy and of cancer cells, respectively, at time $t$ and with resistance phenotype expression $x$, is a nonlocal Lotka-Volterra-like integro-differential model:

$$
\left\{\begin{array}{l}
\frac{\partial}{\partial t} n_{H}(x, t)=\left(\frac{r_{H}(x)}{1+\alpha_{H} u_{2}(t)}-d_{H}(x) I_{H}(t)-u_{1}(t) \mu_{H}(x)\right) n_{H}(x, t) \\
\frac{\partial}{\partial t} n_{C}(x, t)=\left(\frac{r_{C}(x)}{1+\alpha_{C} u_{2}(t)}-d_{C}(x) I_{C}(t)-u_{1}(t) \mu_{C}(x)\right) n_{C}(x, t)
\end{array}\right.
$$

In these equations, $r_{[H, C]}(x)$ and $d_{[H, C]}(x)$ are the proliferation rate (velocity of the division cycle) and the death rate (apoptosis rate) in the two populations. Cytotoxic drugs (cell-killing molecules $u_{1}(t)$ ) are supposed to additively increase the death rate (term $u_{1}(t) \mu_{H}(x)$ ), whereas cytostatic drugs (non cell-killing, but slowing down the cell cycle, molecules $u_{2}(t)$ ) are supposed to multiplicatively decrease the intrinsic cell division velocity (term $\left.\frac{1}{1+\alpha_{[H, C]} u_{2}(t)}\right)$ without affecting the apoptosis rate. Furthermore, we assume differentiability of functions with respect to the phenotypic structuring variable $x, r_{[H, C]}, d_{[H, C]}$ and $\mu_{[H, C]}$, with $\mu^{\prime}()<$.0 (decreasing effects of 
cytotoxic drugs with increasing resistance), $r^{\prime}()<$.0 and $d^{\prime}()>$.0 (cost of resistance paid in terms of proliferation, which ecologists call 'evolutionary double bind'). Independently of the action of drugs, nonlocal logistic terms $I_{H}(t)=a_{H H} \int_{0}^{1} n_{H}(t, x) d x+a_{H C} \int_{0}^{1} n_{C}(t, x) d x$ and $I_{C}(t)=a_{C H} \int_{0}^{1} n_{H}(t, x) d x+a_{C C} \int_{0}^{1} n_{C}(t, x) d x$ (with $a_{H C}$ and $a_{C H}$ small w.r.t. $a_{H H}$ and $a_{C C}$ ) stand for intraspecific and interspecific competition for space or nutrients in the populations in presence. It is also possible, as in [10] to introduce as a gradient term an adaptation drift for fast emergence of resistance, together with non-genetic instability as a Laplacian. Such additions in the model induce fast modifications of the resistance phenotype, representing Lamarckian adaptation, biologically relying on epigenetic mechanisms.

Optimisation of combined anticancer treatments. In this setting, it is natural to use optimal control methods of combined cytotoxic and cytostatic drugs to at least contain the cancer cell population while preserving the healthy cell population, and this involves control of the resistant subpopulation, as it comes induced by cytotoxic drug pressure. It is indeed possible to show analytically that the best therapeutic strategy consists in letting the population evolve under cytostatic containment, with no cytotoxic drug at all for a long time, thus allowing it to concentrate around a sharp, close-to-Dirac resistance phenotype, and after waiting for this as late as possible, then hit it by cytotoxic insult at the maximum possible dose. So that in these settings, the optimal control procedure is to use neither maximum tolerated dose (MTD) of cytotoxic initially, nor metronomic therapy (regularly spaced small doses [11]), but rather 'do not shoot on the enemy until you can see the white of their eyes', i.e., hit with a cytotoxic drug when the distribution of the resistance phenotype in the target cell population is narrowly concentrated around one point [12], see Fig. 1.
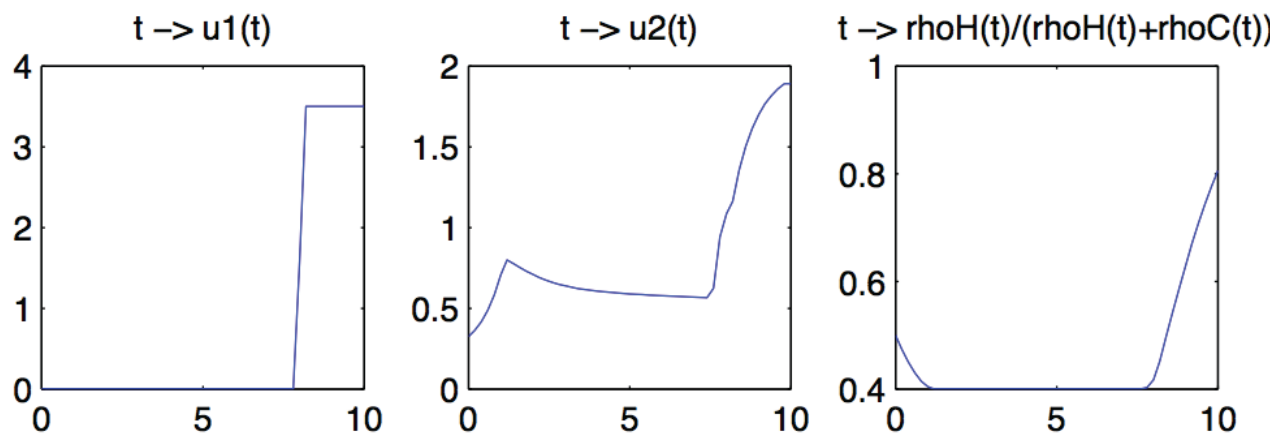

FIGURE 1. Solution to the optimal control problem on system (1), using a combination of the 2 drugs, cytotoxic $u_{1}(t)$ and cytostatic $u_{2}(t)$, the objective function consisting in minimising the number of cancer cells at the end of treatment time under the constraint of constantly preserving the proportion of healthy cells over a predetermined threshold (right panel). The control is singular w.r.t. the cytostatic drug $u_{2}(t)$ and bang-bang w.r.t. the cytotoxic drug $u_{1}(t)$. The first low-dose part of the control law brings the population of cancer cells to a concentrated resistance phenotype, more efficiently destroying it in the second part [12].

Note that hitting a phenotypically dispersed population might result in a branching process splitting an initially indistinct population into sensitive and resistant subpopulations, the latter eventually giving rise to an unbeatable subpopulation, a phenomenon biologically interpretable as "What does not kill me strengthens me" [8]. However, to represent such occurrences, the model (1) is not adapted; a mutational formalism, as in [13] would then be preferrable.

\section{Innovative biological theoretical frameworks}

Cancer and the atavistic hypothesis: diachronic, Darwinian evolution. According to the atavistic hypothesis [14], cancer is in the perspective of Darwinian evolution a regression from sophisticated, finely regulated multicellular organisms with epigenetic regulations on top of it all, controlling differentiation processes that are essential to multicellularity, that themselves control the basic mechanisms of life that are proliferation and programmed death. Losing the most evolved regulation mechanisms drives cancer cell populations to uncontrolled proliferation (the basic mechanism of life), while conserving for their own benefit - and not for the entire organism - in a dispersed order (de)differentiation and plasticity mechanisms that they will be able to use in stress situations such as drug insult.

The metaphor of the epigenetic landscape: synchronic Lamarckian adaptation, or Waddington revisited. In the complementary modern view of the epigenetic landscape [7, 15], cells endowed with a given genome stochastically choose and follow deterministic 'valleys' that normally converge, determined by a quasi-potential of differentiation, to final mature states; but in cancer differentiation is arrested at an early stage, being trapped in local attractors that are forbidden in healthy differentiation. In these local, physiologically unused quasi-potential wells, they proliferate 
without control, remaining in an immature state. Note that this process has nothing to do with Darwinian evolution, it is stochastic exploration of the epigenetic landscape (in a modern sense, epigenetically determined, see Fig. 2).
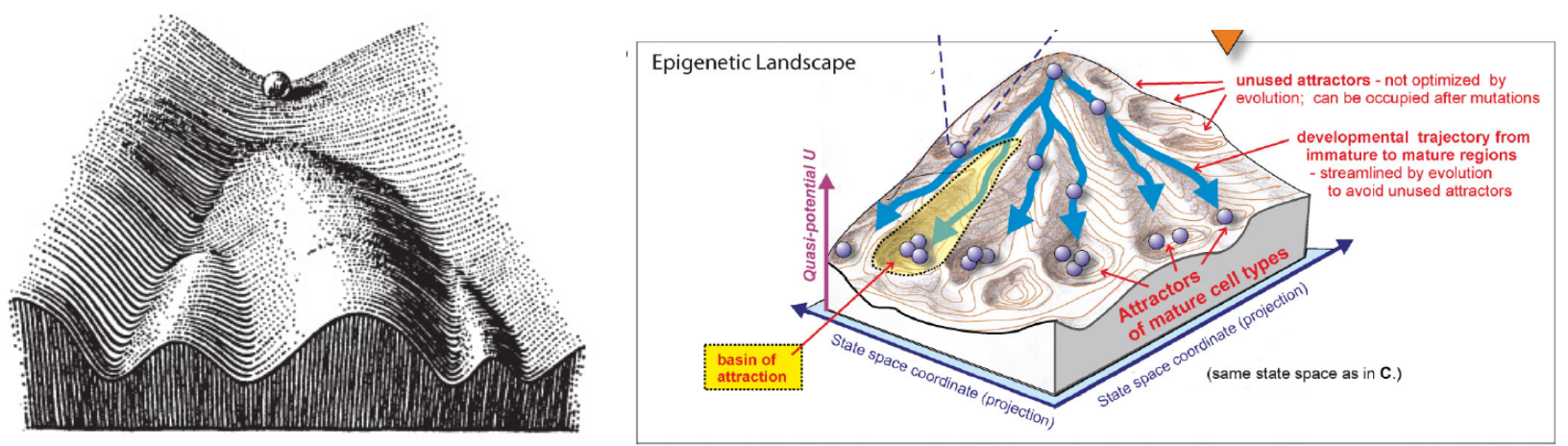

FIGURE 2. The epigenetic landscape was originally introduced by Waddington (left, version of 1957) as a metaphor of physiological cell differentiation, relying on stochastic choices in a deterministic environment, represented as valleys eventually converging in the process of differentiation from a totipotent status to different stable mature states of cells. Revisited by Stuart Kauffman and Sui Huang [7], the same epigenetic landscape now (right, taken from [15] with permission) includes representation of cancer states as local quasi-potential wells - in which immature cells are trapped - that already existed in the physiological landscape, but had been barred in the process of cell differentiation, as evolutionary dead-ends in the process of Darwinian evolution and selection. Note that the epigenetic landscape is not a fitness landscape. See the rich literature of S. Huang's team for details.

These two complementary views about evolution and cancer clearly do not give the solution to curing cancer. However, the innovative perspective they offer orients towards 'targetting the weaknesses' of cancer cell populations [14], which may consist of fighting their plasticity (epigenetic drugs presently trigger high interest from pharmaceutical companies), of designing immunotherapies (using the immune response, a mechanism on top of evolution in multicellular organisms, inaccessible to tumour populations), but not of using only cytotoxic drugs, for it is precisely in proliferation that cancer cell populations are the strongest.

\section{REFERENCES}

1. J. H. Goldie, and A. J. Coldman, Cancer Treat Rep 63, 1727-1733 (1979).

2. P. Savage, J. Stebbing, M. Bower, and T. Crook, Nat Clin Pract Oncol 6, 43-52 (2009), URL http://dx. do i . org/10 . $1038 /$ ncponc1260.

3. L. Ding, T. Ley, D. Larson, C. Miller, D. Koboldt, J. Welch, J. Ritchey, M. Young, T. Lamprecht, M. McLellan, et al., Nature 481, 506-510 (2012).

4. S. V. Sharma, D. Y. Lee, B. Li, M. P. Quinlan, F. Takahashi, S. Maheswaran, U. McDermott, N. Azizian, L. Zou, M. A. Fischbach, et al., Cell 141, 69-80 (2010), ISSN 0092-8674, URL http://dx.doi.org/10.1016/j.cel1.2010. 02.027.

5. F. Delhommeau, S. Dupont, V. Della Valle, et al., N Engl J Med 360, 2289-2301 (2009), URL http: / / dx . doi . org/ 10 . $1056 /$ NEJMOa0810069.

6. T. Haferlach, Hematology Am Soc Hematol Educ Program pp. 400-411 (2008), URL http: //dx. doi . org/10.1182/ asheducation-2008.1.400.

7. S. Huang, Cancer Metastasis Rev 32, 423-448 (2013), URL http: / / dx . doi .org/10.1007/s10555-013-9435-7.

8. A. O. Pisco, and S. Huang, Br J Cancer 112, 1725-1732 (2015), URL http://dx.doi.org/10.1038/bjc.2015. 146.

9. B. J. Druker, and N. B. Lydon, J Clin Invest 105, 3-7 (2000), URL http: / / dx. doi .org/10.1172/ JCI 9083.

10. R. H. Chisholm, T. Lorenzi, A. Lorz, A. K. Larsen, L. N. de Almeida, A. Escargueil, and J. Clairambault, Cancer Res $\mathbf{7 5}$, 930-939 (2015), URL http: / / dx . doi.org/10.1158/0008-5472. CAN-14-2103.

11. E. Pasquier, M. Kavallaris, and N. André, Nat Rev Clin Oncol 7, 455-465 (2010), URL http://dx.doi.org/10 . 1038 /nrclinonc.2010.82.

12. E. Trélat, A. Lorz, and J. Clairambault, Optimal therapy in cancer treatment (2015), work in progress.

13. M. Delitala, and T. Lorenzi, Discrete and Continuous Dynamical Systems - Series B 18, 2267-2282 (2013), ISSN 1531-3492, URL http://dx.doi.org/10.3934/dcdsb.2013.18.2267.

14. C. H. Lineweaver, P. C. W. Davies, and M. D. Vincent, Bioessays 36, 827-835 (2014), URL http: //dx.doi.org/10. 1002 /bies. 201400070.

15. S. Huang, Bioessays 34, 149-157 (2012), URL http: //dx.doi.org/10.1002/bies. 201100031. 